\title{
Geometric engineering of quantum field theories
}

\section{Citation}

Katz, Sheldon, Albrecht Klemm, and Cumrun Vafa. 1997. "Geometric Engineering of Quantum Field Theories." Nuclear Physics B 497 (1-2): 173-95. https://doi.org/10.1016/ s0550-3213(97)00282-4.

\section{Permanent link}

http://nrs.harvard.edu/urn-3:HUL.InstRepos:41385073

\section{Terms of Use}

This article was downloaded from Harvard University's DASH repository, and is made available under the terms and conditions applicable to Other Posted Material, as set forth at http:// nrs.harvard.edu/urn-3:HUL.InstRepos:dash.current.terms-of-use\#LAA

\section{Share Your Story}

The Harvard community has made this article openly available.

Please share how this access benefits you. Submit a story.

\section{Accessibility}


hep-th/9609239

EFI-96-37

HUTP-96/A046

OSU-M-96-24

\title{
Geometric Engineering of Quantum Field Theories
}

\author{
Sheldon Katz ${ }^{1}$, Albrecht Klemm ${ }^{2}$ and Cumrun Vafa ${ }^{3}$ \\ ${ }^{1}$ Department of Mathematics \\ Oklahoma State University \\ Stillwater, OK 74078, USA \\ ${ }^{2}$ Enrico Fermi Institute \\ University of Chicago \\ Chicago, IL 60637, USA \\ ${ }^{3}$ Lyman Laboratory of Physics \\ Harvard University \\ Cambridge, MA 02138, USA \\ katz@math.okstate.edu, aklemm@maxwell.uchicago.edu, vafa@string.harvard.edu
}

Using the recent advances in our understanding of non-perturbative aspects of type II strings we show how non-trivial exact results for $N=2$ quantum field theories can be reduced to T-dualities of string theory. This is done by constructing a local geometric realization of quantum field theories together with a local application of mirror symmetry. This construction is not based on any duality conjecture and thus reduces non-trivial quantum field theory results to much better understood T-dualities of type II strings. Moreover it can be used in principle to construct in a systematic way the vacuum structure for arbitrary $N=2$ gauge theories with matter representations.

September 1996 


\section{Introduction}

In the past few years string duality has taught us many important lessons about nonperturbative aspects of string theory. In each case where there is a reasonable duality conjecture, it often requires certain non-perturbative corrections or non-trivial solitonic spectrum for the duality to work. As an example, type IIA (K3) duality with heterotic $\left(T^{4}\right)$ requires that the $A D E$ singularities of $K 3$ lead to enhanced gauge symmetry of $A D E$ type [1]. This has been subsequently verified by realizing that 2-branes of type IIA wrapped around vanishing 2-cycles lead to precisely the missing states expected for gauge symmetry enhancement (generalizing the observation of [2] [3]). This has been analyzed in detail in [4] [5].

In retrospect we do not need the postulate of duality to derive the existence of enhanced gauge symmetry, even though that was helpful in raising the question of physical interpretation of ADE singularity of type IIA compactification on $K 3$. This is the case with many similar examples and thus we can actually state that independently of string duality conjectures, which despite massive evidence to support them are still conjectural, the existence of solitons and their properties are on much firmer grounds and we can claim to have understood how to deal with them in string theory. This has become much firmer thanks to the very simple description of D-branes as the relevant source for RR charge [6]. A manifestation of this understanding of solitonic objects in string theory is the ability to count the appropriate D-brane states and account for extremal black hole entropy which is not predicted by any known string duality [7].

Certain non-perturbative results for quantum field theories has been derived using string dualities. A particular class of such theories involve $N=2$ supersymmetric theories in $d=4$ where type II on CY 3-fold is dual to heterotic on $K 3 \times T^{2}$ [8] [10]. These dualities fit well with our understanding of how gauge symmetry arises in type II theories due to the observation [11] that the main examples in [8] involve $K 3$ fibered $\mathrm{CY}$ and the adiabatic argument [12] reduces the mechanism of the gauge symmetry enhancement to the case of $K 3$. This aspect of it is in fact emphasized in deriving the field theory consequences of these dualities [13] [14].

The question we wish to address in this paper is whether we can derive non-trivial field theory results directly as a consequence of the recently acquired deeper understanding of string theory dynamics, rather than as a result of a consequence of a duality conjecture. If so we can claim to understand non-trivial results in field theory simply based on the 
existence of string theory and its established properties! As we shall see this will lead directly not only to a stringy confirmation of conjectured results in field theory but also to potentially new results in field theory. An interesting arena to test these ideas is in the case of $N=2$ supersymmetric theories in $d=4$, which is what we will focus on in this paper, even though we believe our methods should be generalizable to other cases and in particular to $N=1$ theories in $d=4$ (for some results in this directions see in particular [15]).

In an $N=2$ field theory one starts with a gauge group $G$, with matter in some representation $\oplus R_{i}$ of $G$. The mass for matter can be incorporated by hand, or more naturally by considering $G$ to have some $U(1)$ factors under which the $R_{i}$ are charged, and by going to the Coulomb phases of the $U(1)$ factors.

Our basic strategy is to find a situation in type IIA theory where exactly the same gauge theory and matter content arise. More precisely, since the gauge symmetries and matter arise near a singular limit of type IIA compactification, and sending $M_{\text {planck }} \rightarrow \infty$ corresponds to studying the local part of the singularity, we do not have to find a global description of type IIA compactification; a local description of the singularity suffices. $N=$ 2 supersymmetry in $d=4$ arises for type IIA compactification on Calabi-Yau threefold. So we are looking for a local model where a Calabi-Yau singularity gives rise to the requisite gauge group $G$ with the matter content $R_{i}$. As for the gauge group, given that it arises in 6 dimensions once we have $A D E$ singularities, if we fiber that over some surface we could easily obtain $A D E$ gauge symmetry in 4 dimensions [16] [17] [18], or by using outer automorphisms [19] we can even realize non-simply laced gauge groups in 4 dimensions [19]20. In particular if we have a genus $g$ curve with $A D E$ singularity one expects to have $A D E$ gauge symmetry in 4 dimensions with $g$ adjoint hypermultiplets [17]. If we are interested in asymptotically free theories we would want to have the genus of the curve be $g \leq 1$. The case $g=1$ will lead to $N=4$ spectrum. So we will consider the $g=0$ case.

Other matter representations can also be obtained from more intricate singularities [4] 21] 20] 22]. In particular if we have an $A D E$ singularity over a surface and at some points along the surface the singularity is enhanced to a higher one, then this is equivalent to some matter localized at those points [22]. For example if we have an $A_{n-1}$ singularity in the fiber which is enhanced to $A_{n}$ at $k$ points on the surface we end up getting $S U(n) \times U(1)$ gauge symmetry with $k$ hypermultiplets in the fundamental of $S U(n)$ charged under $U(1)$ [22]. This spectrum can be understood locally as breaking of the adjoint of $S U(n+1)$ to 
$S U(n) \times U(1)$ at $k$ points on the surface, each leading to a hypermultiplet. Other examples of gauge groups with various matter representations have been discussed in [22].

The basic organization of this paper is as follows. In section 2 we start our discussion of the setup for the simple example of $S U(2)$ without matter and the limit of turning off gravity. In section 3 we discuss quantum correction (or lack thereof) in this context and the relation of worldsheet instantons to pointlike spacetime instantons. In section 4 we review some facts about mirror symmetry applied in a local sense. Even though mirror symmetry is well known, the local application we have in mind has not been fully developed (except in some special cases, e.g. some universal properties of CY-threefolds admitting flops were discussed in 23] [24], other examples appeared in [25] 24]); we remedy this in section 4. In section 5 we return to the case of $S U(2)$ without matter and show how we can use mirror symmetry results and arrive at the field theory results of Seiberg and Witten [26] [27]. In section 6 we discuss some generalizations including $S U(n)$ without matter and some examples with matter. We hope to return to a more complete list of examples in a forthcoming work [28].

\section{2. $S U(2)$ without matter: an illustrative example}

To obtain an $S U(2)$ gauge symmetry we need an $\mathbf{A}_{1}$ singularity in $K 3$. This means that we need a vanishing 2-sphere $\mathbf{P}^{1}$. The $W^{ \pm}$will correspond to 2-branes wrapped around $\mathbf{P}^{1}$ with two opposite orientations. The mass of $W^{ \pm}$is proportional to the area of $\mathbf{P}^{1}$. To obtain an $N=2$ theory in four dimension we need to fiber this over another 2 -sphere $\mathbf{P}^{1}$. The number of ways this can be done is parameterized by an integer $n$ corresponding to the Hirzebruch surfaces $\mathbf{F}_{n}$. The simplest case is $\mathbf{F}_{0}=\mathbf{P}^{1} \times \mathbf{P}^{1}$. All the $\mathbf{F}_{n}$ in the limit of vanishing Kähler class of the fiber $P^{1}$ are expected to give rise to $S U(2)$ gauge symmetry in $d=4$ with $N=2$ supersymmetry without any matter. Note that $1 / g^{2}$ is proportional to the area of the base $\mathbf{P}^{1}$ because of the compactification from 6 to 4 , where $g$ is the bare gauge coupling constant defined at the string scale.

\subsection{The $M_{\text {planck }} \rightarrow \infty$ limit}

To decouple gravitational and stringy effects and recover pure field theory results all we have to do is to consider a limit discussed in [13]. In particular the bare coupling constant $g$ at the string scale should go to zero if the string scale is being pushed to infinity, simply by running of the gauge coupling constant and asymptotic freedom. Thus 
we take a limit $t_{b} \rightarrow \infty$ where $t_{b}$ denotes the Kähler class of the base. On the other hand in order for us to have a finite $W^{ \pm}$mass in this limit we have to take the area of the fiber $\mathbf{P}^{1}$ which in string units corresponds to mass $\left(W^{ \pm} / M_{\text {string }}\right) \rightarrow 0$, we thus have $t_{f} \rightarrow 0$, where $t_{f}$ denotes the Kähler class of the fiber. Clearly the two limits are not unrelated. In particular in the weak coupling regime the running of the coupling constant is dominated by

$$
\frac{1}{g^{2}} \sim \log \frac{M_{W}}{\Lambda}
$$

which implies that we should consider the limit where

$$
t_{b} \sim-\text { const. } \log t_{f}
$$

We can be more precise. The gauge theory instanton number $n$ will be weighted with $\exp \left(-n / g^{2}\right)$. On the other hand from field theory we know that each power of instanton is accompanied by $1 / a^{4}$ where $a$ is the Cartan expectation value of the $S U(2)$ adjoint, which is proportional to $t_{f}$. Putting these together we thus look for the limit

$$
\begin{gathered}
\exp \left(-1 / g^{2}\right)=\exp \left(-t_{b}\right) \sim \epsilon^{4} \Lambda^{4} \\
t_{f} \sim \epsilon a
\end{gathered}
$$

as $\epsilon \rightarrow 0$. In the following we often set the scale $\Lambda=1$.

\section{Quantum corrections}

So far our description of the Coulomb branch of $N=2$ theories in terms of Kähler classes of Calabi-Yau manifolds in type IIA strings has been purely classical. In the context of strings we can have two types of corrections to the classical result: worldsheet corrections and quantum string corrections. As is well known the Kähler moduli of CalabiYau compactifications do receive corrections due to worldsheet instantons. However thanks to mirror symmetry this is well under control and can be computed exactly, at least for a wide range of cases. As far as stringy quantum corrections to Kähler moduli are concerned they are absent, since the coupling constant field of type IIA is in a hypermultiplet and the Kähler moduli belong to vector multiplets and thus do not talk with each other [29][30], and so we can take the limit of weak string coupling to argue that string tree level should be exact. Thus the only corrections to the classical description we need to worry about are the worldsheet instantons. 
In order to interpret the worldsheet corrections we need to recall some basic facts about compactifications of type IIA on $K 3$. In type IIA compactification on $K 3$ there is an important field equation induced in 6 dimensions [31]

$$
d *(\exp (-2 \phi)) H=\operatorname{tr} R \wedge R-\operatorname{tr} F \wedge F
$$

The case we are considering in 4 dimensions is obtained by considering a fibration of this case over another $\mathbf{P}^{1}$. Let us consider a worldsheet instanton wrapped $n$ times around the base $\mathbf{P}^{1}$ at a given point $x$ in the uncompactified spacetime. If we surround the point $x$ by a 3 -sphere in spacetime and integrate, by definition of the wrapping of the sphere we learn that

$$
\int_{S^{3}} *(\exp (-2 \phi)) H=n
$$

Because of (3.1) this means that we should identify worldsheet instantons which wrap $n$ times around the base as corresponding to point-like gauge theory instantons, with instanton number $n$. Note that we are ignoring the $\int R \wedge R$ term above, because in the limit of $M_{\text {string }} \rightarrow \infty$ they are not relevant. That sometimes the worldsheet instantons play the role of gauge theory instantons was anticipated based on string dualities [32] [1]. Our main point in repeating this here is to emphasize that we do not need any input from string duality to make this link. It can be understood purely in the framework of perturbative type IIA string theory.

\subsection{Prepotential of $N=2$ and the worldsheet instantons}

As is well known the contribution to the prepotential of $N=2$ theories is related to the number of worldsheet instantons which itself can be computed using mirror symmetry. Let $C_{i}$ denote a basis for $H_{2}$ and let $d_{n_{i}}$ denote the number of primitive instantons in the Kähler class denoted by $\sum n_{i} C_{i}$, then the prepotential $\mathcal{F}$, which is a function of the Kähler parameters $t_{i}$, satisfies [33]

$$
\partial_{u v w}^{3} \mathcal{F}=\sum_{n_{i}} n_{u} n_{v} n_{w} d_{n_{i}} \frac{\prod_{i} q_{i}^{n_{i}}}{1-\prod_{i} q_{i}^{n_{i}}}
$$

where $q_{i}=\exp \left(-t_{i}\right)$. Once we know what $d_{n_{i}}$ are and what the limit of turning off gravity effects are, as discussed above, we can thus reproduce the field theory results directly. This has the advantage that will allow us not only to identify the global aspects of the Coulomb branch, but also allows us to isolate individual contributions of worldsheet instantons to the gauge field instantons. In particular if we consider the contribution to $\mathcal{F}$ coming from worldsheet instantons wrapping $n$ times around the base we can see how they would lead to instanton number $n$ corrections to the prepotential in the gauge theory system. 


\section{Local mirror symmetry}

In this section we explain our construction of local mirror symmetry. We consider a local neighborhood $X$ of a surface $S$ in a Calabi-Yau threefold. The local geometry will be enough to describe the type IIA string theory compactified on the Calabi-Yau threefold $M$ in certain physically interesting limits, and will be independent of the choice of CalabiYau containing $X$ in this same limit. The type IIA theory will depend on the Kähler parameter of the local geometry, which we call local $A$ model, in a very complicated way due to worldsheet instantons corrections. We will therefore introduce a mirror description of the local geometry, which we refer to as local $B$ model. Physical quantities of interest in this paper are exactly given in terms of integrals over a meromorphic form in the mirror geometry. In particular we are interested in the cases when the local model for $S$ contains vanishing cycles so that in the limit particle spectra with interesting gauge symmetries and matter content become massless.

Our construction can be given equivalently in the context of the linear sigma model 23] [34] or in the context of toric geometry [35.

\subsection{The local $A$ model}

We will construct the local model for the Kähler geometry as gauged $N=2$ two dimensional linear sigma model [23] with $n+3$ chiral multiplets $X_{i}$ which are charged under a $G=U(1)^{n}$ gauge group. The charges of the fields $X_{i}$ are grouped into charge vectors $v^{(k)}=\left(q_{1}^{(k)}, \ldots, q_{n+3}^{(k)}\right)$. Non anomalous $R$-symmetry of the field theory is equivalent to triviality of the canonical bundle in the neighborhood $X$ and implies $\sum_{i=1}^{n+3} q_{i}^{(k)}=0$. To get the space of classical vacua we analyze the zero locus of the scalar potential $U$. The latter is in absence of a superpotential just given by the $D$ terms

$$
U=\sum_{k=1}^{n} \frac{1}{2 e_{k}^{2}} D_{k}^{2}, \quad \text { with } D_{k}=-e_{k}^{2}\left(\sum q_{i}^{(k)}\left|x_{i}\right|-r_{k}\right) \text {, }
$$

where the $r_{k}$ are to be identified with the Kähler parameters, and describes in our case a three dimensional variety.

To illustrate this we consider a theory with $U(1) \times U(1)$ gauge group and charges $v^{(1)}=(1,1,-2,0,0), v^{(2)}=(0,0,1,-2,1)$. The scalar potential reads

$$
U=\frac{e_{1}^{2}}{2}\left(\left|x_{1}\right|^{2}+\left|x_{2}\right|^{2}-2\left|x_{3}\right|^{2}-r_{1}\right)^{2}+\frac{e_{2}^{2}}{2}\left(\left|x_{3}\right|^{2}-2\left|x_{4}\right|^{2}+\left|x_{5}\right|^{2}-r_{2}\right)^{2}
$$


In the phase where $r_{1}$ and $r_{2}$ are both positive, 1 we see that for our vacua we cannot have $x_{1}=x_{2}=0$ or $x_{3}=x_{5}=0$. The set of these fields modulo gauge equivalence is parameterized by a local threefold $X$ with trivial canonical bundle. The space $X$ contains a surface $S$ defined by $x_{4}=0$, which is in fact the Hirzebruch surface $F_{2}$ that is a ruled surface over $\mathbf{P}^{1}$ with fiber $\mathbf{P}^{1}$. Thus the non-compact Calabi-Yau manifold $X$ has a compact part which we identify with $F_{2}$ and the total space $X$ can be identified with $F_{2}$ together with the normal bundle on it (which is identified with the canonical line bundle for $F_{2}$ given here by the $x_{4}$ direction). Our model resolves a curve of $A_{1}$ singularity parameterized by $\mathbf{P}^{1}$; the fibers of $F_{2}$ are the vanishing cycles. The well-known cohomology of $F_{2}$ is generated by the class $s$ of a section with $s^{2}=-2$ and the class $f$ of a fiber. The other intersection numbers are $s \cdot f=1$ and $f^{2}=0$. There is another section $H=s+2 f$ which is disjoint from $s$. The section $s$ itself is defined by $x_{3}=x_{4}=0$. The curves $x_{1}=x_{4}=0$ and $x_{2}=x_{4}=0$ are fibers $f$ of the Hirzebruch surface. The section $H$ is identified with $x_{4}=x_{5}=0$. The divisors $x_{i}=0$ for $i=1,2,3,5$ are noncompact divisors in $X$ which intersect $S$ in the respective curves $f, f, s, H$. For this reason, we will sometimes refer to these divisors as $f, f, s, H$. The divisor $S$ restricts to $S$ itself as the canonical class $K=K_{S}$ by the adjunction formula.

Starting from the geometry we can find the correct charge identification as follows. We identify Kähler classes on $F_{2}$ with curves, and choose as a basis the classes of $s$ and fiber $f$ (which generate the Mori cone of $F_{2}$ ). Identifying each of these with a $U(1)$, we have to find the charges of the fields under $G$. The fields $x_{i}$ are identified with divisors $x_{i}=0$ on our local model $X$. The charge of a field under a $U(1)$ is identified with the intersection number of the divisor associated to the field with the curve representing the $U(1)$. We choose divisors in the order $f, f, s, K, H$. Using the formula $K=-2 s-4 f$, the charges under the first $U(1)$ are identified with the intersection numbers of the 5 divisors with $s$, while the charges under the second $U(1)$ are identified with the intersection numbers of the 5 divisors with $f$.

The identical situation can be described and clarified somewhat by toric geometry. We seek a 3 dimensional toric variety $X$ containing $F_{2}$ as a divisor. As a toric variety, $F_{2}$ has 4 edges; there must also be a fifth edge associated to the divisor $F_{2}$ in $X$. This is why

1 The $U(1)$ generators were chosen so that the phase we are interested in corresponds to requiring $r_{1}$ and $r_{2}$ to be positive - this is tantamount to choosing a basis for the Mori cone, as we will see. 
5 fields were needed in our model. The calculation concludes as before. The charge vectors $(1,1,2,0,1)$ and $(0,0,1,-2,1)$ are identified with the Mori vectors of $F_{2}$ in this context.

Now consider a local $A$ model arising from compactifying an appropriate string theory on a Calabi-Yau hypersurface in a toric variety $V$ associated to a reflexive polyhedron $\Delta$. Such a theory has a linear sigma model analogue which has $k$ additional fieldse $X_{i}$ and $k-1$ additional $U(1)$ gauge factors but since it includes a superpotential, which leads to a constraint, its vacuum configuration is of the same dimension. Suppose that our local model $X$ considered above is contained in $V$ which in particular means that the fields $X_{i}$ are part of the fields for the compact linear sigma model as well. Put in the toric language [35], we suppose that the fan of $X$ is generated by some of the integral points of $\Delta$. In terms of the linear sigma model, this means that after restricting to a subset of 5 of the fields of the full theory, two charge vectors can be found which coincide with the vectors $(1,1,-2,0,0),(0,0,1,-2,1)$.

\subsection{The local B model}

The data of the local $B$ model as we will see gives rise to a complex variety of dimension 2 less than $X$ (Riemann surface for the case of CY 3-folds) and a meromorphic form $\Omega$ on it. Physical quantities in the appropriate limit of the Calabi-Yau threefold are exactly given by periods of $\Omega$ on this Riemann surface which we denote by $\hat{S}$. It turns out that $\hat{S}$ is obtained as a hypersurface in the projectivization of a more natural 3-fold mirror which we denote by $\hat{X}$.

We start by constructing $\hat{X}$ following a method of Batyrev-Borisov [35], which has an equivalent formulation in terms of the linear $\sigma$-model [23] [34].

We introduce variables $y_{i}$ to each field in the linear sigma model. (or equivalently for each edge of the toric fan). Then the $y_{i}$ may be interpreted as coordinates on $\hat{X}$, and for each charge vector $v^{(i)}=\left(q_{1}^{(i)}, \ldots, q_{r}^{(i)}\right)$, the relation

$$
\prod_{q_{j}^{(i)}>0} y_{j}^{q_{j}^{(i)}}=\prod_{q_{j}^{(i)}<0} y_{j}^{-q_{j}^{(i)}}
$$

is satisfied. For example, the quintic has a single charge vector $(-5,1,1,1,1,1)$, and the quintic mirror can be coordinatized by the forms

$$
\left(y_{0}, \ldots, y_{5}\right)=\left(x_{1} \cdots x_{5}, x_{1}^{5}, \ldots x_{5}^{5}\right) .
$$

2 Note that it also contains an additional auxiliary field called $P$ in [23], which is necessary to implement the constraint. 
These satisfy the relation $y_{0}^{5}=y_{1} \cdots y_{5}$ as claimed. In our case we get $n$ equations on the $n+3$ fields $y_{i}$ leaving us with local coordinates $\left(t_{1}, t_{2}, t_{3}\right)$. As we will see below the relevant aspect of the local mirror geometry is given by a projectivisation of the coordinates $\left(t_{1}, t_{2}, t_{3}\right)$. We define $\hat{S}$ therefore as the complex one dimensional hypersurface obtained in projectivizing all the $y_{i}$ 's by

$$
P=\sum_{i=1}^{n+3} a_{i} y_{i}(t)=0
$$

E.g. in the case of our $F_{2}$ model, we get equations

$$
y_{1} y_{2}=y_{3}^{2}, y_{3} y_{5}=y_{4}^{2}
$$

The equations can be solved by putting

$$
\left(y_{1}, \ldots, y_{5}\right)=\left(z, s^{4} / z, s^{2}, s t, t^{2}\right)
$$

Thus $(z, t)$ (defined by projectivizing and setting $s=1$ ) can be taken as local coordinates on $\hat{S}$ subject to the condition

$$
P=a_{1} z+a_{2} \frac{1}{z}+a_{3}+a_{4} t+a_{5} t^{2}=0
$$

In a compact situation the quantum corrected Kähler moduli of a compact CalabiYau $M$ are given by the periods of the holomorphic 3-form on the mirror $\hat{M}$. In our case we have a local part of $M$ denoted by $X$ and we are looking for the worldsheet quantum corrections to it. We have already argued that the local model for the mirror naturally contains a Riemann surface. It is thus natural to expect that the relevant period integrals of $\hat{M}$ are reduced in this limit to period integrals of an appropriate 1-form on the Riemann surface. As we will now show this is indeed the case.

The relevant periods of $\hat{M}$ are governed by the Picard-Fuchs equations which can be derived directly from the charge vectors of the local $A$-model. In the simplest cases they are given by

$$
\prod_{q_{i}^{(k)}>0}\left(\frac{\partial}{\partial a_{i}}\right)^{q_{i}^{(k)}}=\prod_{q_{i}^{(k)}<0}\left(\frac{\partial}{\partial a_{i}}\right)^{-q_{i}^{(k)}}
$$

in terms of the charge vectors, where the $a_{i}$ are the coefficients of the fields in the superpotential of the gauged linear $\sigma$ model of the global $B$-model. As $P$ is actually a restriction 
of this superpotential they are the same coefficients as the ones appearing in (4.3) . In the present context we are giving only a subset of differential equations which are governed by the compact mirror, simply because we only are interested in the Kähler classes which control the size of $X \subset M$. This however implies that no matter what the rest of the data which go into defining the compact version $M$, its periods still satisfy the PF equations (4.6) where the parameters control the Kähler classes of $X$ in $M$.

As an example consider the $F_{2}$ case discussed above. In this case the Picard-Fuchs equations simplify 3 to

$$
\begin{aligned}
& \mathcal{L}_{1}=\theta_{b}{ }^{2}-z_{b}\left(\theta_{f}-2 \theta_{b}\right)\left(\theta_{f}-2 \theta_{b}-1\right) \\
& \mathcal{L}_{2}=\theta_{f}\left(\theta_{f}-2 \theta_{b}\right)-z_{f}\left(2 \theta_{f}\right)\left(2 \theta_{f}+1\right)
\end{aligned}
$$

where $\theta_{i}=z_{i} \partial / \partial z_{i}$ and we have introduced the invariant coordinates $z_{b}=a_{1} a_{2} / a_{3}^{2}, z_{f}=$ $a_{3} a_{5} / a_{4}^{2}$ on the moduli space of $\hat{M}$. More generally we define one $z$ for each charge vector by the relation $z_{k}=\prod a_{i}^{q_{i}^{(k)}}$. The crucial point is that these operators are independent of the choice of $(M, \hat{M})$ which contains our local $A$ - and $B$-models.

We are thus left with the task of finding a way of solving these PF equations. It is in this way that the Riemann surface we constructed above becomes useful, as we will now see. We will define a 1 -form $\Omega$ on the Riemann surface whose periods solve the relevant $\mathrm{PF}$ equations for the mirror.

Note that after solving the equation for constraints of $y_{i}$ we are left with three independent generators $y_{i}$. Moreover since we are interested in the projectivization we can consider only a pair of them by going to the inhomogeneous coordinates. Let $y_{1}$ and $y_{2}$ be these two variables. We define $\Omega$ as follows:

$$
\Omega=\log P \frac{d y_{1}}{y_{1}} \wedge \frac{d y_{2}}{y_{2}} .
$$

Note that this form is well defined up to an addition independent of the moduli. This ambiguity in the shift is reflected by the fact that in the systems we consider 1 is always a solution to the PF equations. This obviously fulfills the Picard-Fuchs equation (4.6) and is a function of $z_{i}$. The derivatives of this form

$$
a_{i} \frac{\partial}{\partial a_{i}} \Omega=\frac{a_{i} y_{i}}{P} \frac{d y_{1}}{y_{1}} \wedge \frac{d y_{2}}{y_{2}}
$$

3 We will use here the same conventions as in 36]

4 The periods $F$ of interest are scale invariant, in the sense that $F(t a)=F(a)$ where $a=$ $\left(a_{1}, \ldots, a_{5}\right)$. This is already clear from our discussion of the differential operators, since $F$ is a function of $z_{1}, z_{2}$. This freedom allows us to set $s=1$ in 4.4 to get $\left(z, 1 / z, 1, t, t^{2}\right)$ which are already visible as the monomials which describe the Seiberg-Witten $S U(2)$ curve. We will make this more precise in the next section. 
give by performing the residue integral around $P=0$ forms on the Riemnann surface which are unambiguous and among which are the holomorphic ones. This can be viewed, alternatively, as rigorizing the definition of the form $\Omega$ given above.

Next we like to see that (4.8) reduces to the meromorphic one form on the general Riemann surfaces for $N=2$ gauge theories we construct from toric data.

To see this we partially integrate one coordinate and then perform the integration over $\frac{d P}{P}$

$$
\begin{aligned}
\int \log P \frac{d y_{1}}{y_{1}} \wedge \frac{d y_{2}}{y_{2}} & =\int \log P d \log y_{1} \wedge \frac{d y_{2}}{y_{2}} \\
& =-\int \log y_{1} \frac{d P}{P} \wedge \frac{d y_{2}}{y_{2}} \\
& =-\int \log y_{1} \frac{d y_{2}}{y_{2}}
\end{aligned}
$$

\subsection{Other $F_{n}$ examples}

As discussed previously if we have any $F_{n}$ surface sitting in the CY 3-fold, in a suitable limit where the fiber shrinks and the base grows, we expect an $S U(2)$ gauge symmetry. Above we showed how we can geometrically 'engineer' the $F_{2}$ inside a Calabi-Yau. Let us now illustrate this engineering for two more cases $F_{0}=\mathbf{P}^{1} \times \mathbf{P}^{1}$ and $F_{1}$. In the next section we will check that all these cases will lead to the same results in the field theory limit, as expected.

We first consider $S=\mathbf{P}^{1} \times \mathbf{P}^{1}$. There are two different fibrations in this case; we denote the respective fibers by $f_{1}$ and $f_{2}$. The intersection numbers are $f_{1}^{2}=f_{2}^{2}=0, f_{1} \cdot f_{2}=1$. Realizing $S$ as a toric variety, we have 4 edges, corresponding to divisors with cohomology classes $f_{1}, f_{1}, f_{2}, f_{2}$ (the fibers over 0 and $\infty$ for each fibration). As in the $F_{1}$ case, there is another toric divisor in the local model $X$, namely the divisor $S$, which restricts to $K=K_{S}=-2 f_{1}-2 f_{2}$. The generators of the Mori cone are $f_{1}$ and $f_{2}$. Ordering the divisors as $K, f_{2}, f_{2}, f_{1}, f_{1}$, we get the charge or Mori vectors $(-2,1,1,0,0),(-2,0,0,1,1)$ and the equations

$$
\begin{aligned}
& \theta_{z_{1}}^{2}-z_{1}\left(2 \theta_{z_{1}}+2 \theta_{z_{2}}\right)\left(2 \theta_{z_{1}}+2 \theta_{z_{2}}+1\right)=0 \\
& \theta_{z_{2}}^{2}-z_{2}\left(2 \theta_{z_{1}}+2 \theta_{z_{2}}\right)\left(2 \theta_{z_{1}}+2 \theta_{z_{2}}+1\right)=0
\end{aligned}
$$

with $z_{1}=a_{2} a_{3} / a_{1}^{2}$ and $z_{2}=a_{4} a_{5} / a_{1}^{2}$.

Our third example is $S=F_{1}$. Its cohomology is generated by a section $s$ with $s^{2}=-1$ and a fiber $f$. The other intersection numbers are $s \cdot f=1$ and $f^{2}=0$. As a toric variety, 
$S$ has another toric divisor which is another section $H$ in the class $s+f$. In this case, we have $K=-2 s-3 f$. Choosing the divisors in the order $K, H, f, s, f$ and identifying the $U(1)$ factors with the Mori generators $s, f$, we compute the charge vectors to be $(-1,0,1,-1,1),(-2,1,0,1,0)$. We put $z_{1}=a_{2} a_{4} /\left(a_{0} a_{3}\right)$ and $z_{2}=a_{1} a_{3} / a_{0}^{2}$. We derive the equations

$$
\begin{gathered}
\theta_{z_{b}}^{2}-z_{b}\left(-\theta_{z_{b}}-2 \theta_{z_{f}}\right)\left(-\theta_{z_{b}}+\theta_{z_{f}}\right)=0 \\
\left(\theta_{z_{f}}\right)\left(-\theta_{z_{b}}+\theta_{z_{f}}\right)-z_{f}\left(-\theta_{z_{b}}-2 \theta_{z_{f}}\right)\left(-\theta_{z_{1}}-2 \theta_{z_{f}}-1\right)=0 .
\end{gathered}
$$

In the limit as the fibers of $F_{0}, F_{1}$ or $F_{2}$ shrink, we get an $S U(2)$ enhancement of the gauge symmetry. In the next section, we show from the local Picard-Fuchs equations that they lead to the same physics in the limit, as expected.

\section{5. $S U(2)$ with no matter revisited}

We now return to the example we started in the paper namely the case where we expect $S U(2)$ without any matter, e.g. $F_{0}\left(=\mathbf{P}^{1} \times \mathbf{P}^{1}\right), F_{1}$ and $F_{2}$. To begin with, the prepotential will be a function of two Kähler classes $t_{b}, t_{f}$ corresponding to the base and fiber respectively. Let $d_{n, m}$ denote the number of primitive worldsheet instantons wrapping $n$ times around the base and $m$ times around the fiber. Let us first consider the prepotential in the absence of gauge theory instantons. As discussed before this should correspond to contributions from worldsheet instantons with zero winding around the base. The relevant instanton numbers to compute are $d_{0, m}$. We have

$$
d_{0,1}=-2, \quad d_{0, i}=0, \quad \forall i>1
$$

This is easy to see. We choose a point on the base and wrap a curve around the fiber $\mathbf{P}^{1}$. There is only one such $\mathbf{P}^{1}$, so apart from the multicover we have only the $d_{0,1} \neq 0$. Since we can choose any point on the base to wrap around the $\mathbf{P}^{1}$ we have the base $\mathbf{P}^{1}$ family of degree 1 maps. The corresponding 'number of instantons' in this case is simply the characteristic class on this moduli space which is $c_{1}\left(\mathbf{P}^{1}\right)=-2$.

We now simply consider the instanton expansion of the three point coupling in which the three points of the sphere are mapped to the divisor dual to the fiber, i.e. $\partial_{t_{f}}^{3} \mathcal{F}$. After integrating this w.r.t $t_{f}$ it should reproduce in the double scaling limit discussed in section (2.1) exactly the running coupling of the field theory $\partial_{a}^{2} \mathcal{F}=-i \tau=\frac{4 \pi}{g^{2}}+2 \pi i \theta$. 
Let us consider the contribution to the gauge coupling constant which is encoded in the second derivative of the prepotential, which is

$$
\partial_{t_{f}}^{2} \mathcal{F}=\sum_{n=0}^{\infty} \sum_{\substack{m=1 \\ k=1}}^{\infty} d_{n, m} m^{2} \frac{q_{b}^{n k} q_{f}^{m k}}{k}
$$

If we now consider the perturbative limit, i.e. concentrate on the terms in the above expansion with $n=0$, and use the value of $d_{0, m}$ noted above we find

$$
\partial_{t_{f}}^{2} \mathcal{F}_{\text {pert }}=-i \tau_{\text {pert }}=-2 \sum_{k=1}^{\infty} \frac{q_{f}^{k}}{k}=2 \log \left(1-q_{f}\right)
$$

As discussed in section 2, we need to consider the limit

$$
q_{f}=\exp \left(-t_{f}\right) \rightarrow \exp (-\epsilon a) \rightarrow 1-\epsilon a
$$

Thus we obtain in this limit we obtain the running coupling constant $-i \tau_{\text {pert }}=2 \log (a)+$ const. $+O(\epsilon)$. That is, the logarithm of the field theory 1-loop diagram comes from the single type holomorphic curve wrapping once the fiber plus its multicover contributions. Aspects of this correspondence was already noted in [17] in the context of gauge theories with asymptotically non-free matter. Much more generally for curves wrapping around the components of the fibers which are sphere trees of $A D E$-type the contributions from the single types of curves will likewise resum to the perturbative part of the $N=2$ coupling constants with $A D E$ gauge symmetry. This is essentially clear because the worldsheet instantons which wrap only around the fiber wrap around individual $\mathbf{P}^{1} \mathrm{~s}$ and thus reproduce the sum of log terms one for each $\mathbf{P}^{1}$ which are in one-to-one correspondence with the positive roots of the gauge group.

Now we come to the more interesting corrections corresponding to the gauge theory instantons, which correspond to world sheet instantons wrapping around the base. Let us now see what is expected in the field theory limit. In this case we are taking the base to be large, and in particular $q_{b} \rightarrow \epsilon^{4}, q_{f} \rightarrow 1-\epsilon a$. This in particular means that in this limit the multi-cover contributions of worldsheet instantons are suppressed, because they are in the denominator in the combination $\left(1-q_{b}^{n} q_{f}^{m}\right) \rightarrow 1$. Ignoring the multicover contribution allows us to write the $\partial_{t_{f}}^{2} \mathcal{F}$ in a simple way

$$
-i \tau=\partial_{t_{f}}^{2} \mathcal{F} \rightarrow \sum_{n, m} d_{n, m} m^{2} q_{b}^{n} q_{f}^{m}
$$


In the field theory limit we expect that the instanton number $n$ contribution to $\tau$ goes as $1 / a^{4 n}$. Let us see how such a behavior may emerge from the above sum. In the limit $q_{f} \rightarrow 1$ only the asymptotic growth of the above sum is relevant. Let us assume that $d_{n, m} \sim \gamma_{n} m^{\alpha(n)}$ for large $m$. Then the leading contribution to $\tau$ from gauge theory instanton number $n$ gives a term proportional to

$$
q_{b}^{n} \sum m^{2+\alpha(n)} q_{f}^{m} \propto \frac{q_{b}^{n}}{\left(1-q_{f}\right)^{3+\alpha(n)}} \propto \frac{\epsilon^{4 n}}{(\epsilon a)^{3+\alpha(n)}}
$$

we thus expect, in order to obtain a non-trivial field theory limit as $\epsilon \rightarrow 0$, that $\alpha(n)=$ $4 n-3$, i.e. we expect that

$$
d_{n, m} \sim \gamma_{n} m^{4 n-3}
$$

for fixed $n$ in the limit of large $m$. Moreover we expect that $\gamma_{n}$ should not depend on how we realize the gauge theory system, i.e. we should get the same result for all $\mathbf{F}_{n}$ (up to some trivial overall rescaling of $a$ ).

Let us inspect the number of instantons for the first few cases, using the local mirror symmetry discussed in section 4 . For gauge theory instanton number one, which corresponds to worldsheet instantons wrapping once around the base and arbitrary number of times around the fiber the numbers grows linearly. For example we have

$$
d_{1, m}^{F_{0}}=-(2 m+2), \quad d_{1, m}^{F_{1}}=2 m+1, \quad d_{1, m}^{F_{2}}=-2 m
$$

This is in accordance with the expected growth given in (5.2). Also notice that the coefficient of the growth $\gamma_{1}$ is the same in all these cases (the fact that they differ by a sign for odd instanton numbers for $F_{1}$ is a check on the relation of $F_{1}$ with a discrete theta angle discussed in [37]). At $n=2$ one observes $d_{2,0}^{F_{1}}=d_{2,0}^{F_{2}}=0$ and $d_{2, i}^{F_{0}}=d_{2, i+1}^{F_{1}}=d_{2, i+1}^{F_{2}}$ where the number for $d^{F_{0}}$ appear in the following table. The numbers for $F_{0}$ and $F_{2}$ for higher $m$ are just shifted $d_{n, m}^{F_{2}}=d_{n, m-n-1}^{F_{0}}$, with $d_{n, m}^{F_{2}}=0$ for $m-n-1<0$. The numbers for $F_{1}$ for higher $n$ are not reported. They differ from the $F_{0}$ and $F_{2}$ cases but have a similar growth. 


\begin{tabular}{|r|rrrrr|}
\hline$m$ & $d_{0, m}^{F_{0}}$ & $d_{1, m}^{F_{0}}$ & $d_{2, m}^{F_{0}}$ & $d_{3, m}^{F_{0}}$ & $d_{4, m}^{F_{0}}$ \\
\hline 1 & -2 & -4 & -6 & -8 & -10 \\
2 & 0 & -6 & -32 & -110 & -288 \\
3 & 0 & -8 & -110 & -756 & -3556 \\
4 & 0 & -10 & -288 & -3556 & -27264 \\
5 & 0 & -12 & -644 & -13072 & -153324 \\
6 & 0 & -14 & -1280 & -40338 & -690400 \\
7 & 0 & -16 & -2340 & -109120 & -2627482 \\
8 & 0 & -18 & -4000 & -266266 & -8757888 \\
9 & 0 & -20 & -6490 & -597888 & -26216372 \\
10 & 0 & -22 & -10080 & -1253538 & -71783040 \\
11 & 0 & -24 & -15106 & -2481024 & -182298480 \\
12 & 0 & -26 & -21952 & -4675050 & -434054144 \\
13 & 0 & -28 & -31080 & -8443424 & -977304976 \\
14 & 0 & -30 & -43008 & -14695208 & -2095334784 \\
15 & 0 & -32 & -58344 & -24755858 & \\
16 & 0 & -34 & -77760 & & \\
$\vdots$ & & & & &
\end{tabular}

The growth in the instanton number is in accordance with expectations based on field theory (5.2). In the limit we are discussing the precise relation of $\gamma_{n}$ with the corrected prepotential is obtained as follows. In the $\epsilon \rightarrow 0$ limit we have

$$
\partial_{a}^{2} \mathcal{F}=2 \log a-\sum_{n=1}^{\infty} \gamma_{n} \frac{(4 n-1) !}{a^{4 n}}
$$

By comparison with the $N=2$ gauge coupling constant

$$
\tau=\frac{4 i}{\pi}\left(\log \left(\frac{\tilde{a}}{\Lambda}\right)+c-\frac{1}{8} \sum_{n=1}^{\infty}(4 n-2)(4 n-1)\left(\frac{\Lambda}{\tilde{a}}\right)^{4 n} \mathcal{F}_{n}\right)
$$

we can fix the proportionality constant to be $a \sim 2 \cdot 2^{1 / 4} \tilde{a}$ (note that the overall rescaling of $\mathcal{F}$ is not physically relevant). The space-time instantons and the asymptotic growth of the worldsheet instantons are related by

$$
\gamma_{n}=\frac{2^{3(3 n-1)}}{(4 n-3) !} \mathcal{F}_{n}
$$


The $\mathcal{F}_{n}$ can be readily calculated since $\mathcal{F}(a)$ is completely determined by the periods $a(u)=\int_{a} \lambda, \partial_{a} \mathcal{F}(a)=a_{D}=\int_{b} \lambda$ of the meromorphic form $\lambda=\frac{i \sqrt{2}}{4 \pi} 2 x^{2} \frac{d x}{y}$ over the Seiberg-Witten curve [27]

$$
y^{2}=\left(x^{2}-u\right)^{2}-\Lambda^{4}
$$

The first few are $\mathcal{F}_{n}=\frac{1}{2^{5}}, \frac{5}{2^{14}}, \frac{3}{2^{18}}, \frac{1469}{2^{31}} \ldots$ for $n=1,2, \ldots$ The prediction that the number of worldsheet instantons grow asymptotically as $d_{n, m} \sim 2 m, \frac{1}{12} m^{5}, \frac{1}{1890} m^{9}, \frac{113}{119750400} m^{13}, \ldots$ for $n=1,2, \ldots$ is in good agreement with the data in table 1 . Below we will also establish this fact to all orders both geometrically as well as by showing that the differential equation which governs $\mathcal{F}$ goes in a particular limit [13] to the differential equation for $a_{D}, a$ of the Yang-Mills system (5.3).

\subsection{Embedding of the Seiberg-Witten curve.}

Before we consider the Picard-Fuchs equations which govern $\mathcal{F}(a)$ let us see the very simple way in which the Seiberg-Witten curves arise from local mirror symmetry. In the previous section we discussed the $F_{2}$ geometric construction in detail. As discussed in the previous section, the mirror geometry is given by the Riemann surface

$$
P=a_{1} z+a_{2} \frac{1}{z}+b_{2}+b_{1} t+b_{0} t^{2}=0
$$

The good algebraic coordinates, which are invariant under scaling w.r.t the charge vectors are

$$
z_{b}=\frac{a_{1} a_{2}}{b_{2}^{2}}, \quad z_{f}=\frac{b_{0} b_{2}}{b_{1}^{2}}
$$

and we will set $a_{1}=a_{2}=b_{0}=1$ in the following.

Now the only thing we need to know is that the base becomes large with $z_{b} \sim q_{b} \sim$ $e^{-t_{b}} \sim \epsilon^{4}$, which implies that $b_{2} \sim \frac{1}{\epsilon^{2}}$. What remains to complete the moduli identification and taking the limit to the $S U(2)$ elliptic curve is to bring (5.4) in the form used in [38] 114] by getting rid of the next to leading term in $t$ by rescaling $t=\left(\sqrt{2} x-\frac{b_{1}}{2}\right)$. This leeads to

$$
P=z+\frac{1}{z}+2\left(x^{2}-u\right)=0
$$

with $u=\frac{1}{2}\left(b_{2}-\frac{b_{1}^{2}}{4}\right)$ required to be finite. This means $2 \epsilon^{2} u:=\left(1-\frac{1}{4} \frac{1}{z_{f}}\right)$ which gives the precise description of the limit we are taking in the good algebraic coordinates. Identifying $z=y-\left(x^{2}-u\right)$ the equation (5.5) can be brought to the form (5.3). 
Now for the Calabi-Yau threefold case with pure $S U(2)$ we have in our conventions the one-form (4.10)

$$
\int \log (t) \frac{d z}{z}
$$

Using the change of variable $t=\sqrt{2} x-\frac{1}{2} b_{1}$ with $b_{1} \sim \epsilon^{-1}$, in the limit $\epsilon \rightarrow 0$ we get the period of

$$
\int \log \left(x-\mathcal{O}\left(\frac{1}{\epsilon}\right)\right) \frac{d z}{z}=-\log (\mathcal{O}(\epsilon)) \int \frac{d z}{z}+\epsilon \int x \frac{d z}{z}
$$

Note that the residue of the first term around $z=0$ gives the period associated with $S$ field and the next term gives the periods of $a$ and $a_{D}$ which as expected are proportional to $\epsilon$.

\subsection{Specialization of the Picard-Fuchs equations}

Even though what we showed above is sufficient to prove that we obtain the expected $S U(2)$ field theory result, it is helpful to recast the solutions of the periods in terms of the Picard-Fuchs equations. In fact the prepotential of the theory is completely determined by the system of Picard-Fuchs equations.

Due to the the large symmetries in the toric representation of the local mirror geometry the PFs are easy to derive. Together with the straightforward description of the limit above this can be viewed as an effective way of deriving the Picard-Fuchs system for the rigid $N=2$ Yang-Mills theories.

As an example of this consider the case of $\mathbf{P}^{1} \times \mathbf{P}^{1}$ for which we expect to again obtain in the field theory limit $S U(2)$ without matter. Using the PF equations (4.11) and substituting $z_{1} \rightarrow \frac{1}{4 x^{2}}, z_{2} \rightarrow \frac{y}{4}\left(\theta_{1} \rightarrow-\frac{1}{2} \theta_{x}, \theta_{2} \rightarrow \theta_{y}\right)$ one gets a system with discriminant components given by the divisors $x=0, y=0$ and

$$
\Delta=(1-x)^{2}-x^{2} y=0
$$

From the variable identification above it is clear that that the $S U(2)$ point is at a tangential intersection of $\Delta=0$ with $y=0$ and the identification of variables in which we can take the $\epsilon \rightarrow 0$ limit keeping a finite $u$ is forced on us.

Physically we identify $y=e^{-S}=(\epsilon)^{4} \Lambda^{4} e^{-\hat{S}}$ and take the double scaling limit considered in 13

$$
\begin{aligned}
& x_{1}=\epsilon^{2} u=(1-x) \\
& x_{2}=\frac{\Lambda^{2} e^{-\hat{S} / 2}}{u}=\frac{\sqrt{y}}{1-x},
\end{aligned}
$$


to recover in the limit $\epsilon \rightarrow 0$ the pure $S U(2) N=2$ YM at weak coupling. Mathematically $\left(x_{1}=0, x_{2}=0\right)$ describes the normal crossing of $y=0$ and an exceptional divisor and provides variables in which the periods degenerates at worst logarithmically at the boundary of the moduli space.

The indices of the system $\mathcal{L}_{1}\left(x_{1}, x_{2}\right), \mathcal{L}_{2}\left(x_{1}, x_{2}\right)$ (i.e. the shift from an integer of the power growth of the periods in algebraic coordinates) at $\left(x_{1}, x_{2}\right)=0$ are $(0,0)$ and $\left(\frac{1}{2}, 0\right)$ and the solutions are schematically of the form

$$
\begin{gathered}
1 \\
\sqrt{x_{1}}\left(1+\mathcal{O}\left(x_{1}\right)\right)\left(1-\frac{1}{16} x_{2}-\ldots\right)=\epsilon a\left(\frac{\Lambda^{2} e^{-\hat{S} / 2}}{u}\right)\left(1+\mathcal{O}\left(\epsilon^{2} u\right)\right) \\
2 \log \left(x_{1} x_{2}\right)+2 \log \left(1+x_{2}\right)=-S+2 \log \left(1+\epsilon^{2} u\right) \\
\sqrt{x_{1}}\left(1+\mathcal{O}\left(x_{1}\right)\right)\left(\left(1-\frac{1}{16} x_{2}-\ldots\right) \log \left(x_{2}\right)+\mathcal{O}\left(x_{2}\right)\right)=\epsilon a_{D}\left(\frac{\Lambda^{2} e^{-\hat{S} / 2}}{u}\right)\left(1+\mathcal{O}\left(\epsilon^{2} u\right)\right),
\end{gathered}
$$

where $a(u) a_{D}(u)$ are the periods of classical $S u(2)$ Yang-Mills theory. One can easily establish the occurrence of $a, a_{D}$ to all orders in $x_{1}=\frac{\Lambda^{2}}{u}$, by noticing that $\mathcal{L}_{s w}=$ $\lim _{x_{2} \rightarrow 0} \sqrt{\frac{x_{1}}{x_{2}}} \mathcal{L}_{2}\left(x_{1}, x_{2}\right) \sqrt{x_{1} x_{2}}$ is the PF operator for $a, a_{D}$ while $\lim _{x_{2} \rightarrow 0} \sqrt{\frac{x_{1}}{x_{2}}} \mathcal{L}_{2}\left(x_{1}, x_{2}\right) \sqrt{x_{1} x_{2}}$ vanishes on the second and third solution of (5.6).

As a check we wish to show that in the field theory limit we obtain the same PF equations even if we started with other systems which realize pure $S U(2)$. In particular we consider the pure $S U(2)$ Yang-Mills limit of the Picard-Fuchs systems for $F_{1}$ and $F_{2}$. The fact that $F_{2}$ should work follows already from the discussion in section 5.1. However it would also be useful to see how the discriminant loci look even in this case. The story turns out to be very similar to the $F_{0}$ case. For $F_{1}$ one has the system (4.12) and by using the variables $z_{b}=y, z_{f}=\frac{1}{4 x}$ we get the discriminant components $x=0, y=0$ as well as

$$
\Delta_{1}=(1-x)^{2}-x^{2} y+9 x\left(y-\frac{3}{4} y^{2}\right)=0, \quad \Delta_{2}=1-y=0
$$

The tangency between $y=0$ and $\Delta_{1}=0$ is resolved in the in the same way as before $x_{1}=\epsilon^{2} u=(x-1) / 2, \quad x_{2}=\frac{\Lambda^{2} e^{-\hat{s} / 2}}{u}=\frac{\sqrt{-8 y}}{x-1}$ apart from a different rescaling which sets the $u$ parameter to the same scale as before. The indices of the solutions near $\left(x_{1}, x_{2}\right)=(0,0)$, the principal structure of logarithm and the appearance of the Seiberg-Witten periods are exactly the same as it is in (5.6). The third period can be summed up to give now

$$
2 \log \left(x_{1} x_{2}\right)+\frac{1}{2} \log \left(1+\frac{x_{2}}{2}\right)=-S+\frac{1}{2} \log \left(1+\frac{\epsilon^{2} u}{2}\right) .
$$


Away from the $x_{2} \rightarrow 0$ limit the second and fourth periods coincide for the $\mathbf{P}^{1} \times \mathbf{P}^{1}$ - and $F_{1}$-case in many coefficients but not completely.

The $F_{2}$ system (4.7) has discriminant components $z_{b}=0, z_{f}=0$ as well and

$$
\Delta_{1}=\left(1-4 z_{f}\right)^{2}-64 z_{f}^{2} z_{b}=0, \quad \Delta_{2}=1-4 z_{f}=0
$$

After the variable change $x_{1}=\epsilon^{2} u=\left(4 z_{f}-1\right) / 2, \quad x_{2}=\Lambda^{2} e^{-\hat{S} / 2} / u=2 \sqrt{z_{b}} /\left(4 z_{f}-1\right)$ one recovers again the Seiberg-Witten theory for $x_{1}=0$.

\section{Generalizations}

So far we have mainly concentrated on one example, namely $S U(2)$ without matter. This however was just to illustrate the basic idea. Our methods generalize to many other interesting cases. In this section we will first illustrate this in the context of some concrete examples, in particular $S U(n+1)$ without matter and with one fundamental. At the end we will sketch the general idea and what is involved in getting more general gauge groups with matter.

\section{1. $S U(n+1)$ without matter}

Geometrically we have an $A_{n}$ fiber over a base $\mathbf{P}^{1}$. The relevant Kähler parameters appearing in the prepotential are therefore the volume of the base $t_{b}$ and the individual fibers $t_{f_{1}}, \ldots, t_{f_{n}}$ and we are looking at the limit in which the size of the base becomes large, while the one of the fibers are of the same order:

$$
\left(1-q_{f_{i}}\right) \sim \epsilon a_{i}, \quad q_{b} \sim \epsilon^{2 n+2}
$$

By the definition of the $A_{n}$ fiber we have $n$ Kähler classes corresponding to the simple roots of $A_{n}$ and as many -2 spheres as there are positive roots. As discussed before, resuming up the instanton contributions coming from these -2-spheres plus their multicovers gives the perturbative contribution of the prepotential

$$
\mathcal{F}_{1-\text { loop }}(a(u))=\frac{i}{4 \pi} \sum_{\substack{\text { positive } \\ \text { roots } \alpha}} Z_{\alpha}^{2} \log \left[Z_{\alpha}^{2} / \Lambda^{2}\right]
$$

where the $a_{i}$ parameterize vacuum expectation value of the field in the Cartan subalgebra of the gauge group and $Z_{a}=\vec{q} \cdot \vec{a}$. 
The charge vectors defining the local toric variety $X$ describing an $A_{n}$ fibration over $\mathbf{P}^{1}$ are

$$
\begin{aligned}
& v_{b}=(1,1,-2, \quad 0, \quad 0, \quad 0, \quad 0, \ldots, \quad 0, \quad 0, \quad 0, \quad 0) \\
& v_{f_{1}}=(0,0, \quad 1,-2, \quad 1, \quad 0, \quad 0, \ldots, \quad 0, \quad 0, \quad 0, \quad 0)
\end{aligned}
$$

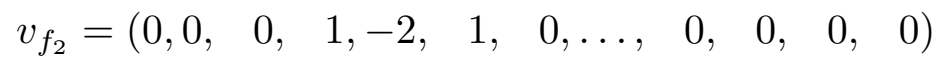

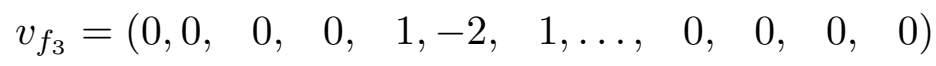

$$
\begin{aligned}
& \vdots \quad \vdots \\
& v_{f_{n-1}}=(0,0, \quad 0, \quad 0, \quad 0, \quad 0, \quad 0, \ldots, \quad 1,-2, \quad 1, \quad 0)
\end{aligned}
$$

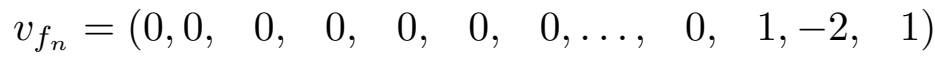

The $n+4$ columns of (6.3) were determined by the procedure described earlier for $S U(2)$ using toric divisors. In this case, the first two are the $A_{n}$ fibers, the next is a divisor meeting the $A_{n}$ surface in a section with self intersection -2 (very much like the divisor $x_{3}=0$ in the $F_{2}$ case). The next $n$ divisors are the $n$ irreducible components of the $A_{n}$ surface, and finally the last divisor meets the $A_{n}$ surface in a disjoint section.

Local mirror symmetry associated to the columns of (6.3) the monomials $z, \frac{1}{z}, 1, t, \ldots t^{n+1}$ which satisfy the constraints required by the charge vectors. The linear constraint defining the family of curves is given by

$$
P=z+\frac{1}{z}+b_{n+1}+b_{n} t+\ldots b_{1} t^{n}+b_{0} t^{n+1}=0 .
$$

The good coordinates are $z_{b}=\frac{1}{b_{n+1}^{2}}, z_{f_{n-i}}=\frac{b_{i+1} b_{i-1}}{b_{i}^{2}}, i=1, \ldots, n$. From (6.1) we have $b_{n+1} \sim \epsilon^{-n-1}$. The scaling of the the other variables follows by shifting $t=\left(2^{\frac{1}{n+1}} x-\frac{b_{1}}{(n+1)}\right)$ comparing with [38]

$$
P=z+\frac{1}{z}+2 W_{A_{n}}(x, \vec{u})=0
$$

and requiring that all Weyl invariant parameters $u_{i}$ stay finite. Especially that implies that $b_{k} \sim \epsilon^{-k}$ in leading order. For the relation between the parameters $a_{i}$ used in (6.2) and the Weyl invariant parameters $u_{i}$ in $W_{A_{n}}(x, \vec{u})$ see e.g. [39].

The charge vectors $v_{b}, v_{f_{1}} \ldots, v_{f_{n}}$ are sufficient to write down the relevant system of 
differential operators $\mathcal{L}_{b}, \mathcal{L}_{1}$ and $\mathcal{L}_{k}$ for $k=2, \ldots, n$

$$
\begin{aligned}
& \mathcal{L}_{b}=\theta_{b}^{2}-z_{b} \prod_{i=0}^{1}\left(2 \theta_{b}-\theta_{f_{1}}+i\right) \\
& \mathcal{L}_{1}=\left(\theta_{f_{1}}-2 \theta_{b}\right)\left(\theta_{f_{1}}-2 \theta_{f_{2}}\right)-z_{f_{1}} \prod_{i=0}^{1}\left(2 \theta_{f_{1}}-\theta_{f_{2}}+i\right), \\
& \mathcal{L}_{k}=\left(\theta_{f_{k}}-2 \theta_{f_{k-1}}+\theta_{f_{k-2}}\right)\left(\theta_{f_{k}}-2 \theta_{f_{k+1}}+\theta_{f_{k+2}}\right)-z_{f_{k}} \prod_{i=0}^{1}\left(2 \theta_{f_{k}}-\theta_{f_{k-1}}-\theta_{f_{k+1}}+i\right),
\end{aligned}
$$

where $\theta_{f_{l}}$ has to be omitted if $l$ out of the index range $1<l<n$. Further $n(n-1) / 2$ second order differential operators follow by considering combinations of the $v_{f_{i}}$, which correspond to positive roots of $A_{n}$ and $n-1$ by factorisation. At $z_{b}=z_{f_{l}}=0$ the system has a constant solution, corresponding to the fact that the meromorphic form (4.8) has a pole with non vanishing residue, $n+1$ solutions starting with $\log \left(z_{b}\right), \log \left(z_{f_{i}}\right)$ and one solution which is quadratic in the logarithms.

Let us discuss the $S U(3)$ case in more detail. The system (6.6) has in this case besides the universal discriminant $\Delta_{b}=1-4 z_{b}=0$ a principal discriminant

$$
\begin{aligned}
\Delta_{p}= & \left(1-4 z_{f_{1}}-4 z_{f_{2}}+18 z_{f_{1}} z_{f_{2}}-27 z_{f_{1}}^{2} z_{f_{2}}^{2}\right)^{2}- \\
& 8 z_{b} z_{f_{1}}^{2}\left(8-9 z_{f_{2}}\left(8-3 z_{f_{2}}\left(7+4 z_{f_{1}}\right)+6 z_{f_{2}}^{2}\left(2+9 z_{f_{1}}\right)+81 z_{f_{2}}^{3} z_{f_{1}}^{2}\left(2 z_{b}-1\right)\right)\right)=0 .
\end{aligned}
$$

The first line, which survives the $z_{b} \rightarrow 0$ limit, is recognized as the $S U(3)$ discriminant. It is present if four points lie on an edge in a toric diagram, as it is familiar e.g. from strong coupling gauge $S U(3)$ enhancements [17] [18]. Comparing (6.4) with (6.5) we have

$$
v=\frac{2 b_{1}^{2}}{27}-\frac{b_{1} b_{2}}{3}+b_{3}, \quad u=-\frac{2^{1 / 3} b_{1}^{2}}{3}+2^{1 / 3} b_{2}
$$

In the limit $b_{3} \sim \epsilon^{-3}$ we get from this in leading orders

$$
z_{b}=\frac{\Lambda^{6} 3^{4}}{2^{2}} \epsilon^{6}, \quad z_{f_{1}}=\frac{1}{3}+\frac{1}{3^{\frac{2}{3}}} \epsilon^{2} u, \quad z_{f_{2}}=\frac{1}{3}+\frac{1}{3^{\frac{2}{3}}} \epsilon^{2} u+3 \epsilon^{3} v
$$

which when inserted in (6.7) reproduces of course in leading order the rigid $S U(3)$ discriminant

$$
\left(u^{3}-27\left(\Lambda^{3}+v\right)^{2}\right)\left(u^{3}-27\left(\Lambda^{3}-v\right)^{2}\right) \epsilon^{12}+\mathcal{O}\left(\epsilon^{13}\right)
$$


There are various choices of resolution variables to solve the theory near $z_{b}=0 \quad z_{f_{1}}=$ $z_{f_{2}}=\frac{1}{3}$ and to derive from the $\mathcal{L}_{i}$ the rigid $S U(N)$ operators, e.g.

$$
x_{1}=\left(z_{f_{2}}-z_{f_{1}}\right) \sim \epsilon^{3} v, \quad x_{2}=\frac{\sqrt{z_{b}}}{\left(z_{f_{2}}-z_{f_{1}}\right)} \sim \frac{1}{v}, \quad x_{3}=\frac{\left(z_{f_{1}}-\frac{1}{3}\right)^{3 / 2}}{\left(z_{f_{2}}-z_{f_{1}}\right)} \sim \sqrt{\frac{u^{3}}{v^{2}}}
$$

\section{2. $S U(n+1)$ with matter}

The generalization to the $A_{n}$ series with matter is straightforward. The charge vectors for $A_{n}$ with one fundamental matter multiplet $\left(N_{f}=1\right)$ are given by

$$
\begin{aligned}
& v_{s}=(1, \quad 1, \quad 0,-2, \quad 0, \quad 0, \quad 0, \quad 0, \ldots, \quad 0, \quad 0, \quad 0, \quad 0) \\
& v_{f_{1}-E}=(0,-1, \quad 1, \quad 1,-1, \quad 0, \quad 0, \quad 0, \ldots, \quad 0,0, \quad 0, \quad 0) \\
& v_{E}=(0, \quad 1,-1, \quad 0,-1, \quad 1, \quad 0, \quad 0, \ldots, \quad 0, \quad 0, \quad 0, \quad 0) \\
& v_{f_{2}}=(0, \quad 0, \quad 0, \quad 0, \quad 1,-2, \quad 1, \quad 0 \ldots, \quad 0, \quad 0, \quad 0, \quad 0) \\
& v_{f_{3}}=(0, \quad 0, \quad 0, \quad 0, \quad 1,-2, \quad 1, \quad 0 \ldots, \quad 0, \quad 0, \quad 0, \quad 0) \\
& \text { : } \quad \text { : }
\end{aligned}
$$

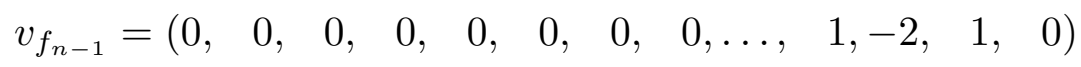

$$
\begin{aligned}
& v_{f_{n}}=(0, \quad 0, \quad 0, \quad 0, \quad 0, \quad 0, \quad 0, \quad 0 \ldots, \quad 0, \quad 1,-2, \quad 1)
\end{aligned}
$$

For $A_{n}=S U(n+1)$, this has $n+5$ columns and $n+2$ rows. The $A_{n}$ surface has $n$ irreducible components, each of which is a ruled surface over $\mathbf{P}^{1}$. These components form a chain as dictated by the $A_{n}$ Dynkin diagram; adjacent components meet along a section of each. chain, the blowup being needed to obtain matter according to [22].

The rows correspond to curves $s, f_{1}-E, E, f_{2}, \ldots, f_{n}$ where $s$ is the -2 curve in the $F_{2}$ at the end of the chain, $f_{i}$ are the individual fibers, and $E$ is the exceptional divisor obtained by blowing up a point of $F_{2}$ ( $E$ lies in the blown up $F_{2}$ and meets the next surface in the chain by choice). The blowup splits a particular $f_{1}$ fiber into two pieces, one of which is $E$ and the other being $f_{1}-E$.

The columns correspond to divisors $f, f-E, E, s, E_{1}, \ldots, E_{n}, H$, where $f$ is a fiber of the entire $A_{n}$ surface (so that $\left.f=\sum f_{i}\right), E_{i}$ are the surfaces comprising the chain, and $H$ is a section of the last surface in the chain which is disjoint from the other surfaces in the chain.

From the charge vectors, we see that we can therefore associate to the rows the monomials $1 / z, z, z t, 1, t, t^{2}, \ldots, t^{n+1}$, so that we just have to add on a single term with a $z t$ to get the matter. 
$N_{f}=2$ can be done similarly — there is a symmetric choice of the blowup (i.e. simply blow up a point on the last component of the chain which meets the next to last component in the chain). The chain has been chosen to start with a blown up $F_{2}$ at the end of the chain, the blowup being needed to obtain matter according to [22].

Let us discuss the $S U(2)$ case with one matter in more detail. In that case we have the charge vectors

$$
\begin{aligned}
v_{b} & =\left(\begin{array}{lll}
1,1, & 0,-2, \quad 0, & 0
\end{array}\right), \\
v_{F-E} & =\left(\begin{array}{lll}
0,-1, & 1, \quad 1,-1, & 0
\end{array}\right), \\
v_{E} & =\left(\begin{array}{lll}
0,1,-1, & 0,-1, & 1
\end{array}\right) .
\end{aligned}
$$

The corresponding relations are fulfilled by the following parameterization of the coordinates of the mirror $z, 1 / z, t / z, 1, t, t^{2}$ which is given again by the linear constraint

$$
P=a_{1} z+a_{2} \frac{1}{z}+a_{3} \frac{t}{z}+b_{2}+b_{1} t+b_{0} t^{2}=0
$$

After the same change of variables as before $t \rightarrow \sqrt{2} x-\frac{1}{2} b_{1}$ and setting $z=y-\left(x^{2}-u\right)$ we arrive at the form [27] of the elliptic curve

$$
y^{2}=\left(x^{2}-u\right)^{2}-\Lambda^{3}(x+m)
$$

with the parameter identification

$$
u=-\frac{1}{2}\left(b_{2}-\frac{1}{4} b_{1}^{2}\right), \quad \Lambda^{3}=\sqrt{2} a_{3}, \quad \Lambda^{3} m=\left(a_{2}-\frac{1}{2} a_{3} b_{1}\right) .
$$

This means that we have to scale $b_{2} \sim \epsilon^{-2}, b_{1} \sim \epsilon^{-1}$ and $a_{2} \sim \epsilon^{-1}$ (we set $a_{1}=a_{3}=b_{0}=1$ in the following). Expressed in the good algebraic coordinates $z_{b}=a_{2} / b_{2}^{2}, z_{F-E}=$ $b_{2} /\left(a_{2} b_{1}\right)$ and $z_{E}=a_{2} / b_{1}$ the limit is

$$
z_{b} \sim \epsilon^{3}, \quad\left(\frac{1}{4} \frac{1}{z_{E} z_{F-E}}-1\right) \sim 2 \epsilon^{2} u, \quad\left(z_{E}-\frac{1}{2}\right) \sim \Lambda^{3} m \epsilon
$$

which is consistent with the expected limit of growing base and shrinking fiber. It has also been checked that the PF system and the discriminant reduce to the one of $N=2 S U(2)$ gauge theory with matter.

It is similarly straightforward to check that the $S U(n+1)$ theory with one matter also reduces to the expected result from field theory. 


\subsection{Main idea in generalizations}

It should be clear from the above examples that whenever we can construct a quantum field theory by 'geometric engineering' of fibered $A D E$ singularities with possibly some extra singularities and at the same time find the PF equations for the dual mirror we would have solved the Coulomb branch of the corresponding quantum field theory. This thus involves 2 basic steps: 1) Construction of the local model; 2) Finding the mirror periods.

To construct the local mirror $\hat{X}$ of a fibered $A D E$ singularity, we first review what we have done in our earlier examples. We first found generators for the Mori cone of the $A D E$ surface. Then we identified certain divisors. These divisors can be described in hindsight without toric geometry. There were two fibers (modified as appropriate if there were blowups). We also required a fiber for each of the irreducible components of the $A D E$ surface. We also required a divisor class for each blowup. Finally, we required two disjoint sections.

In this way, we can take any geometric $A D E$ singularity and any geometric description of extra matter as explained in [22] and now calculate charge vectors from the geometry without using toric geometry directly. The result will generally be a toric description of $X$.

In the best cases, the field $\prod x_{i}$ will be neutral, so that $X$ is itself a toric variety. Then our methods will go through unmodified. We do know that this cannot happen in all examples; but then we can typically construct $X$ as a (noncompact) hypersurface or complete intersection in a toric variety. Then the usual geometric constructions of mirror symmetry can be used to construct $\hat{X}$.

Once we have $\hat{X}$, we can deduce the Picard-Fuchs operators directly from those of compact Calabi-Yau threefolds associated to reflexive polyhedra which 'enlarge' the theory. The scaling behavior of the periods which satisfy these equations is intermediate in complexity between the cases considered above and those arising from reflexive polyhedra. We are optimistic that the scaling behavior will lead us to realize the periods as periods of a meromorphic differential on a curve directly from these techniques.

Acknowledgments: We would like to thank B. Greene, W. Lerche, P. Mayr, D. Morrison, N. Warner and S.-T. Yau for valuable discussions.

The research of SK was partially supported by NSF grant DMS-9311386 and NSA grant MDA904-96-1-0021, that of AK was partially supported by the Clay Fund for Mathematics, through the Department of Mathematics, Harvard University and that of CV was supported in part by NSF grant PHY-92-18167. 


\section{References}

[1] E. Witten, String Theory Dynamics in Various Dimensions, Nucl. Phys. B443 (1995) 85, hep-th/9503124, Some Comments On String Dynamics, Proc. String's 95, hepth/9507121

[2] A. Strominger, Nucl. Phys. B452 (1995) 96, hep-th/9505105

[3] B. Greene, D. Morrison, A. Strominger, Nucl. Phys. B451 (1995) 109, hep-th/9504145.

[4] M. Bershadsky, V. Sadov and C. Vafa,Nucl. Phys. $\underline{B 463}$ (1996) 398, hep-th/9510225.

[5] M. Bershadsky, V. Sadov and C. Vafa, Nucl. Phys. B463 (1996) 420, hep-th/9511222.

[6] J. Polchinski, Dirichlet Branes and Ramond-Ramond Charges, Phys. Rev. Lett. $\underline{75}$ (1995) 4724, hep-th/9510017

[7] A. Strominger and C. Vafa, Phys. Rev. Lett. 77 (1996) 2368

[8] S. Kachru and C. Vafa, Nucl. Phys. B450 (1995) 69, hep-th/9505105.

[9] S. Ferrara, J. Harvey, A. Strominger and C. Vafa, Phys. Lett. B361 (1995) 59 , hepth/9505162.

[10] V. Kaplunovsky, J. Louis, and S. Theisen, Phys. Lett. B357 (1995) 71; A. Klemm, W. Lerche and P. Mayr, Phys. Lett. $\underline{B} 357$ (1995) 313; C. Vafa and E. Witten, preprint HUTP-95-A023; hep-th/9507050; I. Antoniadis, E. Gava, K. Narain and T. Taylor, Nucl. Phys. $\underline{B 455}$ (1995) 109, B. Lian and S.T. Yau, Mirror Maps, Modular Relations and Hypergeometric Series I,II, hep-th/9507151, hep-th 9507153;P. Aspinwall and J. Louis, Phys. Lett. B369 (1996) 233; I. Antoniadis, S. Ferrara and T. Taylor,Nucl. Phys. $\underline{B 460}$ (1996) 489; G. Curio, Phys. Lett. B366 (1996) 131,Phys. Lett. B366 (1996) 78; G. Lopes Cardoso, G. Curio, D. Lüst and T. Mohaupt, Instanton Numbers and Exchange Symmetries in $N=2$ Dual String Pairs, hep-th/9603108

[11] A. Klemm, W. Lerche and P. Mayr, as cited in [10]

[12] C. Vafa and E. Witten, as cited in [10]

[13] S. Kachru, A. Klemm, W. Lerche, P. Mayr and C. Vafa, hep-th/9508155.

[14] A. Klemm, W. Lerche, P. Mayr, C. Vafa, N. Warner, hep-th/9504034.

[15] S. Kachru and E. Silverstein, hep-th/9608194

[16] P. Aspinwall, Phys. Lett. B357 (1995) 329,Phys. Lett. B371 (1996) 231,

[17] S. Katz, D. Morrison and R. Plesser,hep-th/9601108

[18] A. Klemm and P. Mayr, hep-th/9601014

[19] P. Aspinwall and M. Gross, hep-th/9602118

[20] M. Bershadsky, K. Intriligator,S. Kachru, D. Morrison, V. Sadov and C. Vafa, hepth/9605200.

[21] P. Berglund, S. Katz, A. Klemm and P. Mayr, hep-th/9601108

[22] S. Katz and C. Vafa, hep-th/9606086

[23] E. Witten, Nucl. Phys. B403 (1993) 159

[24] P. Aspinwall, B. Greene, D. Morrison, Nucl. Phys. B420 (1994) 184 
[25] A. Klemm, P. Mayr and C. Vafa, hep-th/9607139

[26] N. Seiberg and E. Witten, Nucl. Phys. B426 (1994) 19, hep-th/9407087

[27] N. Seiberg and E. Witten, Nucl. Phys. B431 (1994) 484, hep-th/9408099

[28] A. Klemm, S. Katz and C. Vafa, work in progress.

[29] B. de Wit, P.G. Lauwers, R. Philippe, S.Q. Su, A. van Proeyen, Phys. Lett. B134 (1984) 37; B. de Wit, A. van Proeyen, Nucl. Phys. B245 (1984) 89; J.P. Derendinger, S. Ferrara, A. Masiero, A. van Proeyen, Phys. Lett. B140 (1984) 307; B. de Wit, P.G. Lauwers, A. van Proeyen, Nucl. Phys. B255 (1985) 569; E. Cremmer, C. Kounnas, A. van Proeyen, J.P. Derendinger, S. Ferrara, B. de Wit, L. Girardello, Nucl. Phys. $\underline{B 250}$ (1985) 385

[30] N. Berkovits and W. Siegel, Nucl. Phys. B462 (1996) 213

[31] C. Vafa and E. Witten, Nucl. Phys. B447 (1995) 261

[32] M. Duff and R. Khuri, Nucl. Phys. B411 (1994) 473, C. M. Hull and P.K Townsend, Nucl. Phys. B $\underline{B 438}$ (1995) 109

[33] P. Candelas, X. de la Ossa, P. Green and L. Parkes, Nucl. Phys. B359 (1991) 21

[34] D. Morrison and R. Plesser, Nucl. Phys. B440 (1995) 279, hep-th/9412236, Towards Mirror Symmetry as Duality For Two-Dimensional Abelian Gauge Theories, hepth/9508107

[35] V. Batyrev, Duke Math. Journal 69 (1993) 349, V. Batyrev, Journal Alg. Geom. 3 (1994) 493, V. Batyrev, Quantum Cohomology Rings of Toric Manifolds, preprint 1992, V. Batyrev and A. Borisov, alg-geom/9412017, alg-geom/9509009

[36] S. Hosono. A. Klemm, S. Theisen and S.-T. Yau, Comm. Math. Phys. 167 (1995) 301

[37] S. Katz, M. Douglas and C. Vafa, hep-th/9609071

[38] A. Gorskii, I. Krichever, A. Marshakov, A. Mironov and A. Morozov, Phys. Lett. $\underline{B 355}$ (1995) 466, H. Itoyama and A. Morozov, hep-th/9511126 and hep-th/9512161, E. Martinec and N. Warner,Nucl. Phys. B459 (1996) 97

[39] A. Klemm, W. Lerche and S. Theisen, Int. Journ. Mod. Phys. A11 (1996) 1929, hepth/9505150 